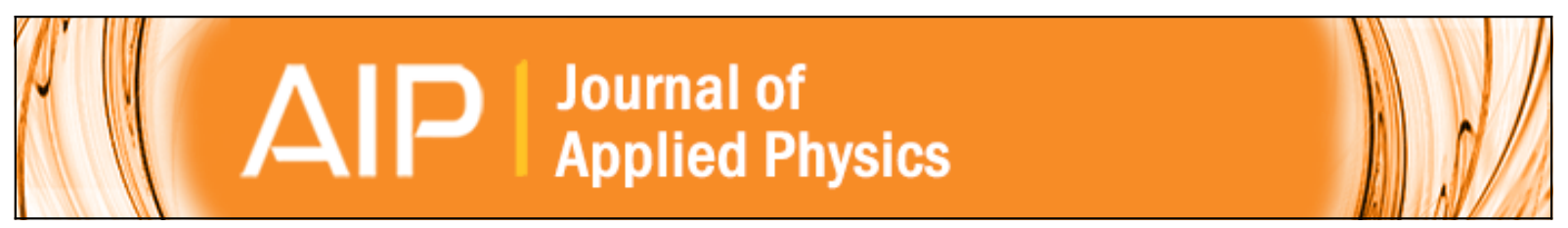

\title{
The effect of cathode geometry on barium transport in hollow cathode plasmas
}

James E. Polk, loannis G. Mikellides, Angela M. Capece, and Ira Katz

Citation: Journal of Applied Physics 115, 183301 (2014); doi: 10.1063/1.4873168

View online: http://dx.doi.org/10.1063/1.4873168

View Table of Contents: http://scitation.aip.org/content/aip/journal/jap/115/18?ver=pdfcov

Published by the AIP Publishing

\section{Articles you may be interested in}

Oxygen transport in the internal xenon plasma of a dispenser hollow cathode

J. Appl. Phys. 115, 153302 (2014); 10.1063/1.4871755

Effect of Ti-Al cathode composition on plasma generation and plasma transport in direct current vacuum arc J. Appl. Phys. 115, 123301 (2014); 10.1063/1.4869199

Transport scaling in interchange-driven toroidal plasmas

Phys. Plasmas 16, 062303 (2009); 10.1063/1.3139261

Hollow cathode theory and experiment. I. Plasma characterization using fast miniature scanning probes J. Appl. Phys. 98, 113302 (2005); 10.1063/1.2135417

Power balance at cathode in glow discharges

Phys. Plasmas 12, 113502 (2005); 10.1063/1.2127929

\section{A|P| $\begin{aligned} & \text { Journal of } \\ & \text { Applied Physics }\end{aligned}$}

Journal of Applied Physics is pleased to announce André Anders as its new Editor-in-Chief 


\title{
The effect of cathode geometry on barium transport in hollow cathode plasmas
}

\author{
James E. Polk, ${ }^{1, \text { a) }}$ Ioannis G. Mikellides, ${ }^{1}$ Angela M. Capece, ${ }^{2}$ and Ira Katz ${ }^{1}$ \\ ${ }^{1}$ Jet Propulsion Laboratory, California Institute of Technology, 4800 Oak Grove Drive, Pasadena, \\ California 91109, USA \\ ${ }^{2}$ California Institute of Technology, 1200 E. California Blvd., Pasadena, California 91125, USA
}

(Received 12 February 2014; accepted 8 April 2014; published online 12 May 2014)

\begin{abstract}
The effect of barium transport on the operation of dispenser hollow cathodes was investigated in numerical modeling of a cathode with two different orifice sizes. Despite large differences in cathode emitter temperature, emitted electron current density, internal xenon neutral and plasma densities, and size of the plasma-surface interaction region, the barium transport in the two geometries is qualitatively very similar. Barium is produced in the insert and flows to the surface through the porous structure. A buildup of neutral $\mathrm{Ba}$ pressure in the plasma over the emitter surface can suppress the reactions supplying the $\mathrm{Ba}$, restricting the net production rate. Neutral Ba flows into the dense Xe plasma and has a high probability of being ionized at the periphery of this zone. The steady state neutral Ba density distribution is determined by a balance between pressure gradient forces and the drag force associated with collisions between neutral $\mathrm{Ba}$ and neutral Xe atoms. A small fraction of the neutral $\mathrm{Ba}$ is lost upstream. The majority of the neutral $\mathrm{Ba}$ is ionized in the high temperature Xe plasma and is pushed back to the emitter surface by the electric field. The steady state $\mathrm{Ba}^{+}$ion density distribution results from a balance between electrostatic and pressure forces, neutral $\mathrm{Xe}$ drag and $\mathrm{Xe}^{+}$ion drag with the dominant forces dependent on location in the discharge. These results indicate that hollow cathodes are very effective at recycling $\mathrm{Ba}$ within the discharge and therefore maintain a high coverage of $\mathrm{Ba}$ on the emitter surface, which reduces the work function and sustains high electron emission current densities at moderate temperatures. Barium recycling is more effective in the cathode with the smaller orifice because the $\mathrm{Ba}$ is ionized in the dense Xe plasma concentrated just upstream of the orifice and pushed back into the hollow cathode. Despite a lower emitter temperature, the large orifice cathode has a higher Ba loss rate through the orifice because the Xe plasma density peaks further upstream. (C) 2014 AIP Publishing LLC. [http://dx.doi.org/10.1063/1.4873168]
\end{abstract}

\section{INTRODUCTION}

Hollow cathode electron emitters are used in electric thrusters for propellant ionization and ion beam neutralization. State-of-the-art hollow cathodes consist of a porous tungsten tube (the "insert") encased in a refractory metal tube. The cathode is preheated using an external resistive heater until the insert reaches the temperature for thermionic emission. Neutral xenon propellant is injected into the cathode and is ionized by electron impact, creating a xenon plasma. Once the discharge is started, the heater is turned off and the insert is heated by ion and/or electron bombardment from the internal plasma. An orifice plate is located downstream of the insert and serves to increase the gas pressure inside the hollow cathode.

A low emitter operating temperature is essential for extending cathode lifetime and is achieved when barium atoms are adsorbed on an oxygen monolayer on top of the tungsten substrate. This forms a $\mathrm{Ba}^{+} \mathrm{O}^{-}$dipole that reduces the work function from $4.5 \mathrm{eV}$ for tungsten to less than $2.1 \mathrm{eV} .{ }^{1}$ In order to maintain this low operating temperature, the tungsten surface must be supplied with $\mathrm{Ba}$ at a rate

a)james.e.polk@jpl.nasa.gov sufficient to balance losses due to evaporation and ion sputtering.

$\mathrm{Ba}$ and $\mathrm{BaO}$ are supplied to the surface by incorporating a barium calcium aluminate source material (the "impregnant") in the pores of the tungsten. Gaseous Ba and $\mathrm{BaO}$ are released in interfacial reactions between the tungsten matrix and the impregnant, producing a temperaturedependent vapor pressure of these species inside the pores. The $\mathrm{Ba}$ and $\mathrm{BaO}$ migrate to the surface by Knudsen flow and surface diffusion on the pore walls. When the barium supply rate from the interior or from the gas phase drops below the rate at which adsorbed atoms are lost from the surface by desorption, the surface coverage drops, and the work function rises. $^{2}$ The cathode eventually becomes impossible to ignite or cannot be heated to the temperatures needed for the required electron current density.

The discharge plasma comprises primarily Xe neutrals, $\mathrm{Xe}^{+}$ions, and electrons. Gas phase barium, oxygen, and tungsten species may also be found in small concentrations. Although the densities of these minor species are small compared with the plasma and Xe neutral densities, they play an important role in the electron emission properties. Long duration cathode tests have shown that tungsten erosion and redeposition in the emission zone resulted in the formation of a dense tungsten shell with reduced porosity. ${ }^{3,4}$ Although 
this inhibited barium flows from the insert pores, cathode performance was unaffected. ${ }^{3,4}$ These observations prompted the development of a Ba transport simulation by Polk et al. ${ }^{5}$ to describe the diffusion of $\mathrm{Ba}$ in the Xe discharge.

Previous simulations were performed for a conventional $6.35 \mathrm{~mm}$ diameter cathode with an insert inner diameter of $3.8 \mathrm{~mm}$ and orifice diameter of $1 \mathrm{~mm}$ at a discharge current of $13.3 \mathrm{~A}$ and a Xe flow rate of $3.7 \mathrm{sccm}^{5}$ These model results showed that Ba supplied by the emitter is recycled in the cathode plasma. Barium flows out of the insert, is ionized in the plasma, and is pushed back to the insert surface by the electric field. The barium deposited on the emitter from the gas phase provides enough coverage to sustain the low work function surface even in regions where the pores may be blocked and barium supply from the interior is inhibited. This study suggested that Ba depletion does not occur as rapidly in plasma cathodes as in vacuum cathodes because of the effect of the plasma in inhibiting reactions and recycling Ba.

In this paper, we extend the transport model to include the effect of finite Xe neutral velocity on Ba diffusion and compare the Ba transport in a cathode with a $2.25 \mathrm{~mm}$ diameter orifice operated at $15 \mathrm{~A}$ and $3.7 \mathrm{sccm}$ to the previous results from a cathode with a $1 \mathrm{~mm}$ diameter orifice. The $1 \mathrm{~mm}$ diameter orifice results in a relatively high internal gas pressure, and a xenon plasma that is concentrated around the entrance to the orifice with electron emission over only the first few millimeters of the insert. The $2.25 \mathrm{~mm}$ diameter orifice yields a much lower gas density and a more extended xenon plasma discharge that draws electron current from a large fraction of the insert length. This paper provides a brief overview of the model and then describes the $\mathrm{Xe}$ and $\mathrm{Ba}$ plasma results from these two simulations. The focus is on the effect that large changes in the Xe plasma conditions have on the barium flow dynamics and resulting emitter surface coverage.

\section{BARIUM TRANSPORT MODEL}

The numerical model of Ba transport has been described in detail previously, ${ }^{5}$ but the major features will be briefly summarized here. The initial focus of the modeling is on Ba because this is the dominant species produced by the insert, ${ }^{6}$ although the model will be extended to include $\mathrm{BaO}$ flow and chemistry. Ba transport is strongly influenced by the Xe plasma-the electron density and temperature control the ionization of $\mathrm{Ba}$, and the electric field in the Xe plasma and collisions with $\mathrm{Xe}$ and $\mathrm{Xe}^{+}$ions control the diffusion of $\mathrm{Ba}$. However, Ba atom and ion densities are so low they do not significantly modify the charge density or temperature in what is primarily a Xe discharge. This allows us to separate the problem into two parts and use the major species parameters predicted by a Xe discharge model developed by Mikellides et al. ${ }^{7}$ as fixed quantities in the solution of the minor species transport equations.

\section{A. Barium flow equations}

The $\mathrm{Ba}$ flow in the insert plasma is modeled using the species momentum and continuity equations. We assume that viscous and magnetic field effects are negligible, that the phenomena of interest are sufficiently slow, i.e., there are no high frequency phenomena and fluid acceleration is small compared to the force terms, and that collisions between minor species (such as $\mathrm{Ba}^{-\mathrm{Ba}^{+}}$collisions) are sufficiently rare that they can be neglected. The flux of minor species $j$ (either $\mathrm{Ba}$ or $\mathrm{Ba}^{+}$), can then be written as the sum of four terms

$$
\begin{aligned}
\Gamma_{j}= & n_{j} \mathbf{u}_{j}=\frac{-\nabla P_{j}}{\nu_{j, X e^{+}} \mu_{j, X e}\left(1+\nu_{j, X e} / \nu_{j, X e^{+}}\right)} \\
& +\frac{e n_{j} \mathbf{E}}{\nu_{j, X e^{+}} \mu_{j, X e}\left(1+\nu_{j, X e} / \nu_{j, X e^{+}}\right)}+n_{j}\left(\frac{1}{1+\nu_{j, X e} / \nu_{j, X e^{+}}}\right) \\
& \times \mathbf{u}_{X e^{+}}+n_{j}\left(\frac{\nu_{j, X e} / \nu_{j, X e^{+}}}{1+\nu_{j, X e} / \nu_{j, X e^{+}}}\right) \mathbf{u}_{X e},
\end{aligned}
$$

where $n_{j}$ is the density of species $j$, $\mathbf{u}_{j}$ is the velocity, $P_{j}$ is the partial pressure, $e$ is the electron charge, $\mathbf{E}$ is the electric field, $\nu_{j, i}$ is the frequency of collisions between species $j$ and $i$, and $\mu_{j, i}$ is the reduced mass. The first two terms represent flux driven by pressure gradient and electrostatic forces. The third term is the flux driven by drag due to collisions with $\mathrm{Xe}^{+}$ions and the fourth is the flux due to drag from collisions with Xe neutrals. Previously, the Xe neutrals were assumed to have negligible flow velocities; ${ }^{5}$ however, in this simulation, we use the values calculated in the Xe discharge model. ${ }^{7}$ For Ba neutrals, the electric field term is zero. The collision frequency terms are cast in the form of ratios to the dominant interaction. The electron drag term is neglected because of the small electron-to-heavy particle mass ratio.

The ion-ion collision frequency that appears in these equations is given by the standard Coulomb frequency, and the neutral Ba-Xe collision frequency is expressed in terms of a constant scattering cross section, $\sigma_{D}=40 \AA^{2}$, based on diffusion measurements for $\mathrm{Ba}$ in $\mathrm{Xe}^{8}{ }^{8} \mathrm{Ba}^{+}-\mathrm{Xe}$ and $\mathrm{Ba}-\mathrm{Xe}^{+}$ collisions are assumed to have the same cross section as Ba-Xe collisions.

The continuity equations for $\mathrm{Ba}$ and $\mathrm{Ba}^{+}$can be expressed in terms of these fluxes

$$
\frac{\partial n_{j}}{\partial t}+\nabla \cdot \Gamma_{j}=\dot{n}_{j},
$$

where $\dot{n}_{B a}=-\dot{n}_{B a^{+}} . \dot{n}_{B a^{+}}$is the rate at which $\mathrm{Ba}^{+}$ions are created by ionization of $\mathrm{Ba}$ neutrals and $\dot{n}_{B a}$ is the rate at which neutrals are consumed by ionization reactions. The ionization rate is given by the product of the $\mathrm{Ba}$ atom density and the ionization rate coefficient, $\dot{n}_{B a^{+}}\left(T_{e}\right)=n_{B a} \nu_{B a}^{i z}$. The ionization rate coefficient for $\mathrm{Ba}$ was obtained by integrating Ba ionization cross sections ${ }^{9}$ over a Maxwellian velocity distribution. The model also employs the ideal gas equation of state. The Ba neutrals and ions are assumed to equilibrate with the Xe atoms and ions, so all heavy particles have a single temperature, $T_{h}$.

The $\mathrm{Ba}$ transport model depends on a number of $\mathrm{Xe}$ plasma parameters, including the Xe atom and ion densities and heavy particle temperature, which appear in the collision frequencies, the electron density and temperature, which 
determine the $\mathrm{Ba}$ ionization rate, and the $\mathrm{Xe}$ neutral and ion velocity and electric field, which appear in the force terms of the momentum equations. These quantities are taken from a separate simulation ${ }^{7}$ of the Xe plasma and are assumed to be independent of the dynamics of the Ba plasma.

The Xe plasma code was modified to solve a discretized form of Eq. (2) on the 2D axisymmetric computational domain and mesh shown in Fig. 1. This region encompasses the insert plasma at the downstream end of the emitter in the hollow cathode. The Ba neutral and ion densities were initially assumed to be a uniform low value, and the continuity equations were then time-marched until the densities and fluxes achieved steady state values.

\section{B. Boundary conditions}

The simulation domain is bounded by five surfaces as shown in Fig. 1, including the centerline, the upstream inlet to the cathode, the entrance to the cathode orifice, the upstream face of the orifice plate, and the emitter surface. Ion and neutral fluxes through these surfaces are specified as boundary conditions.

The centerline is a symmetry boundary, so the ion and neutral fluxes are set equal to zero. The upstream inlet to the cathode is the only major sink for Ba. Ions and neutrals were assumed to flow out of this surface with their thermal velocities and condense on cooler surfaces upstream, as observed experimentally. ${ }^{3,10}$ The upstream face of the orifice plate was assumed to have a steady-state, submonolayer coverage of $\mathrm{Ba}$ with a net neutral flow from the surface equal to the impinging ion flux. Neutrals were assumed to flow out of the cathode orifice with their thermal velocity. Ions were assumed to have the same velocity at this boundary as the $\mathrm{Xe}^{+}$ions. In practice, there are very few $\mathrm{Ba}$ neutrals or ions in this region, so the results are not sensitive to these assumptions.

The emitter surface serves as the source of $\mathrm{Ba}$, which flows from the interior through the porous tungsten. The rate at which $\mathrm{Ba}$ is dispensed depends on insert temperature, $T_{c}$, Ba partial pressure in the insert plasma, $P_{B a}$, and time, $t$. The impregnant decomposition reaction is assumed to be strongly diffusion-limited, so the $\mathrm{Ba}$ pressure at the reaction front is very close to the equilibrium pressure of $\mathrm{Ba}$ over the reactants, which is a function of the insert temperature. The diffusive flow or Knudsen flow through the pores and reaction products in the insert is driven by the difference in the pressure at the reaction front and the $\mathrm{Ba}$ partial pressure at the insert surface. Finally, the reaction front recedes into the insert as the $\mathrm{Ba}$ is consumed, so the flow resistance increases with time. In cases for which the ambient $\mathrm{Ba}$ pressure exceeds the equilibrium pressure, the reaction is assumed to cease. The Ba supply rate has been observed to decrease as $t^{-1 / 2}$ for time $t$, which is consistent with Knudsen flow through a flow resistance which increases as $\mathrm{Ba}$ is lost and the reaction front recedes into the surface. ${ }^{1,11}$ The net $\mathrm{Ba}$ flow is expressed as

$$
\begin{aligned}
\Gamma_{s}\left(T_{c}, P_{B a}, t\right)= & \Gamma_{s}\left(T_{c}, P_{B a}=0, t=t_{r e f}\right) \\
& \times\left(\frac{P_{v}-P_{B a}}{P_{v}}\right)\left(\frac{t_{r e f}}{t}\right)^{1 / 2} .
\end{aligned}
$$

Measurements ${ }^{12}$ of the Ba supply rate from S-type cathodes in vacuum early in life (with a total operating time $t_{\text {ref }}$ on the order of $10 \mathrm{~h}$ ) are used to define the maximum supply rate, $\Gamma_{s}^{\max }$, in atoms $/ \mathrm{cm}^{2} \mathrm{~s}$ as

$$
\Gamma_{s}^{\max }\left(T_{c}, P_{B a}=0, t_{r e f}\right)=\exp 63.53-46237 / T_{c},
$$

where $T_{c}$ is given in $K$. In both cathode geometries, the accumulated operating time was assumed to be $1000 \mathrm{~h}$, so the Ba supply rate was a factor of 10 times lower than the beginning-of-life values ${ }^{12}$ due to recession of the reaction front.

Temperature distributions measured along the axes of the emitters ${ }^{13}$ for the two geometries modeled here are used in the simulations to calculate the maximum barium flow rate as a function of position. The calculated $\mathrm{Ba}$ partial pressure in the insert is used to determine the reduction in barium flow, assuming an equilibrium pressure, $P_{v}$, in Torr for the impregnant decomposition reaction proposed by Rittner $^{11}$

$$
\log P_{v}=8.56-20360 / T_{c} .
$$

Ba neutrals and ions also flow back to the insert surface. Ions enter the sheath at the Bohm velocity, $u_{B}=\sqrt{k T_{e} / m_{B a}}$, and the ion flux is $\Gamma_{B a^{+}}=n_{B a+} u_{B}$. The cathode temperatures are too high for $\mathrm{Ba}$ to accumulate in thick layers on the surface, and the evaporation rate for bulk $\mathrm{Ba}$ at these temperatures exceeds the ion and neutral fluxes by many orders of magnitude, so we assume that the surface has a steady state, submonolayer coverage of $\mathrm{Ba}$ adsorbed on oxygen on tungsten. In steady state, there is no net total Ba flux to the surface. The neutral flux to the surface is balanced by the desorption of

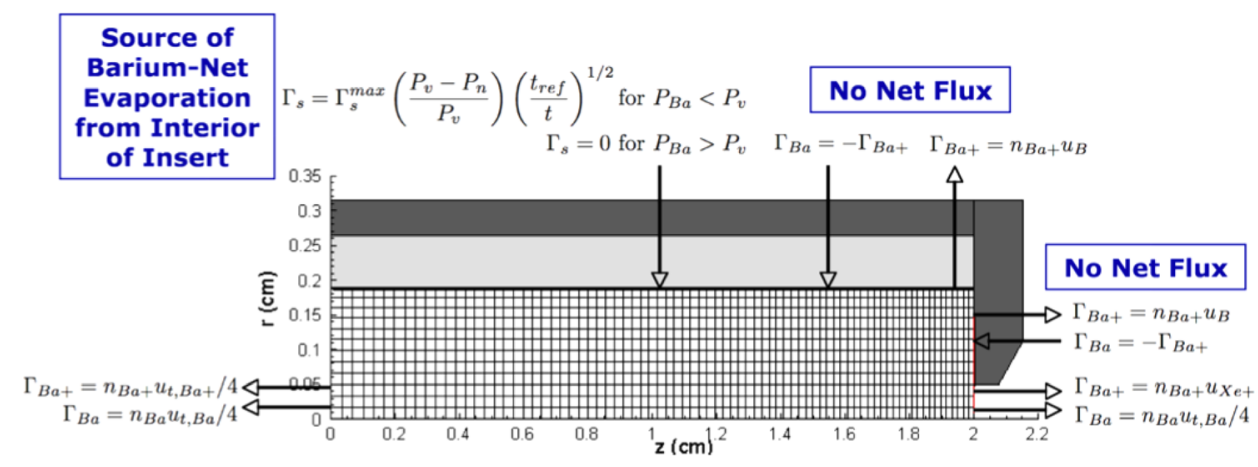

FIG. 1. Boundary conditions and computational grid for the barium transport model. 
neutrals, and ions that strike the surface return to the plasma as neutral $\mathrm{Ba}$ atoms, so there is a net neutral flow (in addition to the neutral flow from insert interior), which is equal in magnitude to the ion flow. This assumption is consistent with the observations from long duration tests that Ba does not accumulate on the downstream surfaces of the inserts. ${ }^{14}$

\section{BARIUM TRANSPORT RESULTS}

The results of the xenon plasma simulation for the $1 \mathrm{~mm}$ diameter orifice cathode at a discharge current of 13.3 A and a xenon flow rate of $3.7 \mathrm{sccm}$ are displayed in Fig. 2, and the results for the cathode with the $2.25 \mathrm{~mm}$ diameter

(a) Xenon neutral density contours, $\mathrm{n}_{\mathrm{xe}} / 10^{21}\left(\mathrm{~m}^{-3}\right)$ and neutral flux streamlines.

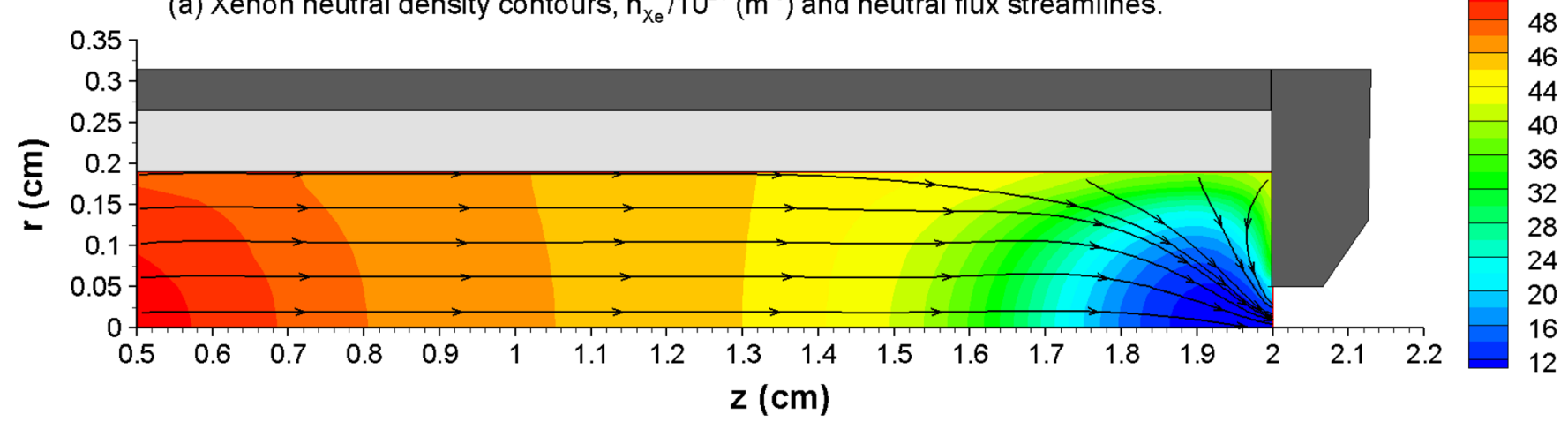

(b) Electron temperature contours, $T_{e}(e V)$ and electron current streamlines.

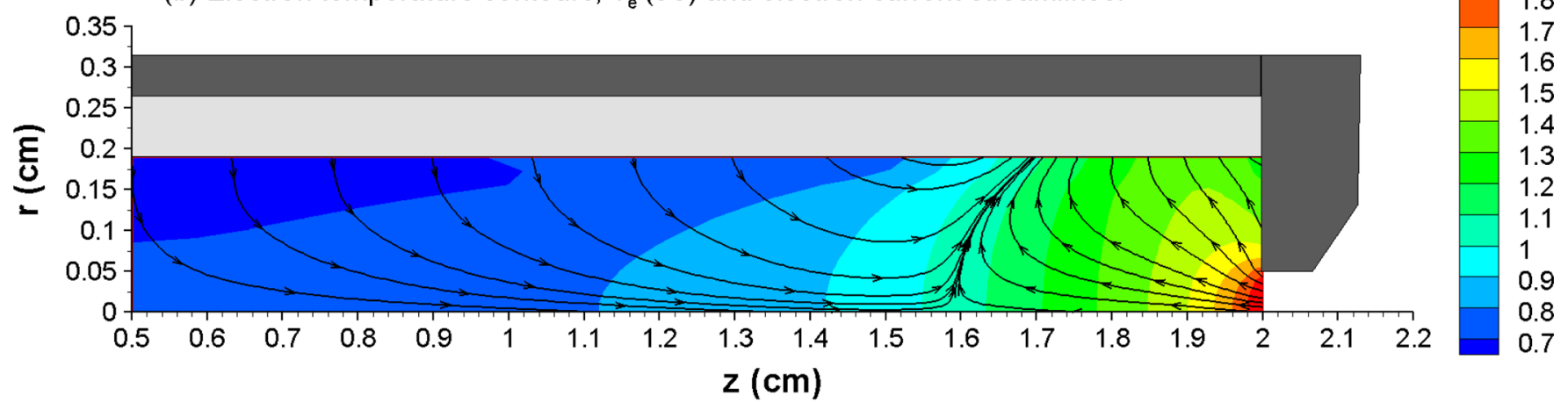

(c) Emitter temperature, $T_{c}\left({ }^{\circ} \mathrm{C}\right)$ and electron emission current density, $j_{e}\left(\mathrm{~A} / \mathrm{cm}^{2}\right)$

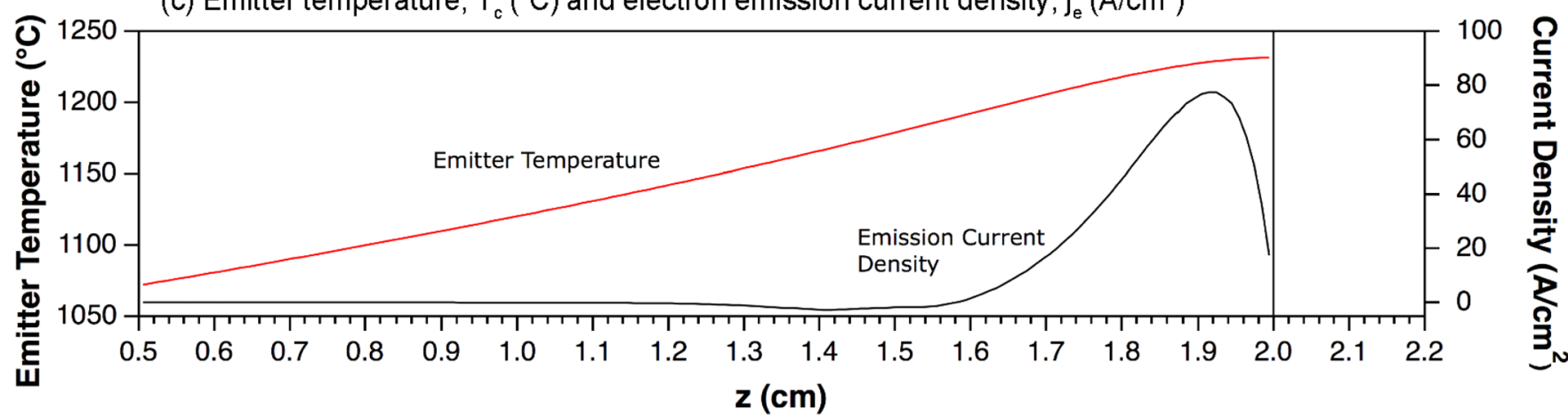

(d) Xenon plasma density contours, $\mathrm{n}_{\mathrm{e}} / 10^{19}\left(\mathrm{~m}^{-3}\right)$ and ion current streamlines.

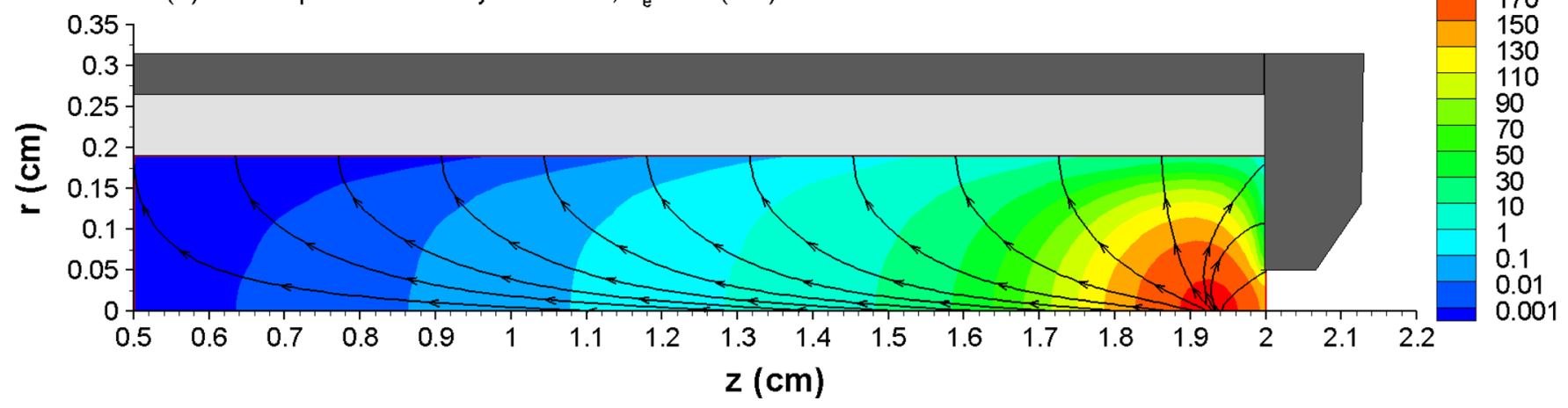

FIG. 2. Xenon plasma solution for the small orifice hollow cathode. 
(a) Xenon neutral density contours, $\mathrm{n}_{\mathrm{Xe}} / 10^{21}\left(\mathrm{~m}^{-3}\right)$ and neutral flux streamlines.

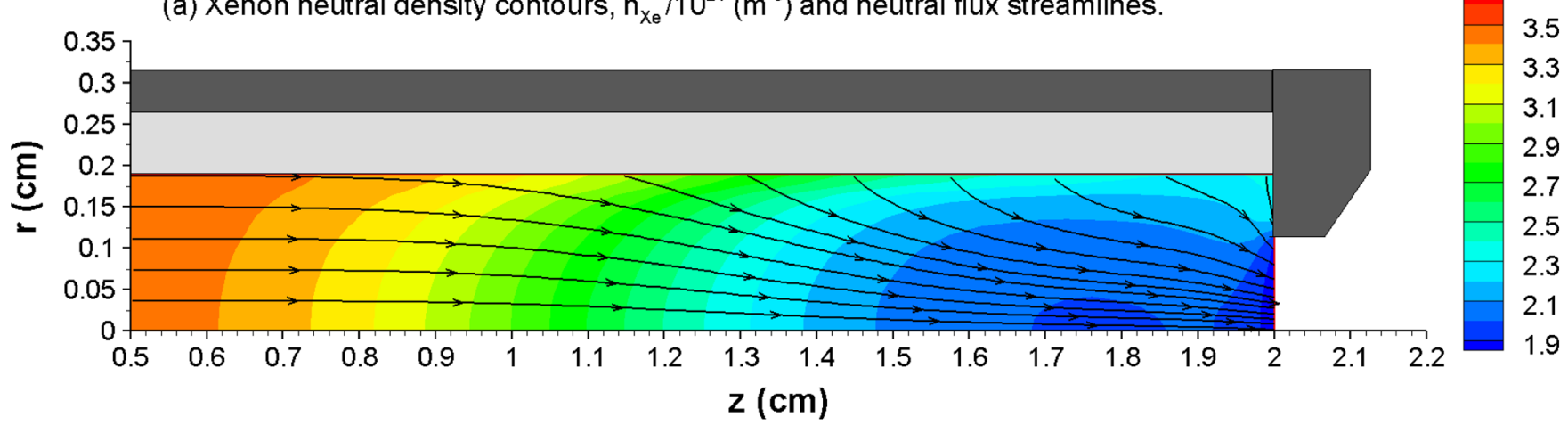

(b) Electron temperature contours, $\mathrm{T}_{\mathrm{e}}(\mathrm{eV})$ and electron current streamlines.

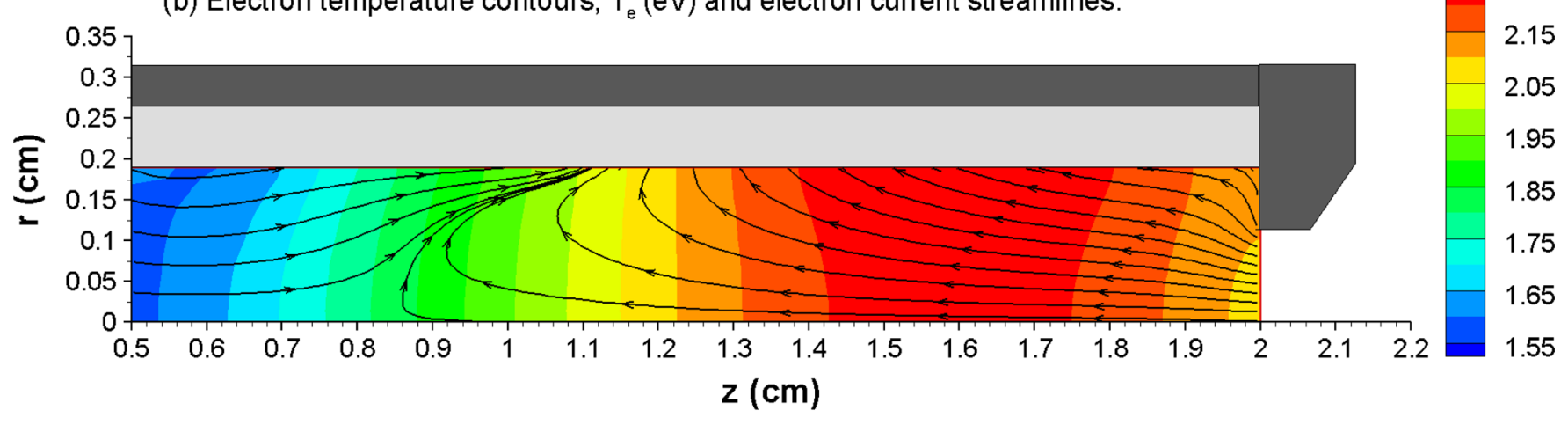

(c) Emitter temperature, $T_{c}\left({ }^{\circ} \mathrm{C}\right)$ and electron emission current density, $j_{\mathrm{e}}\left(A / \mathrm{cm}^{2}\right)$

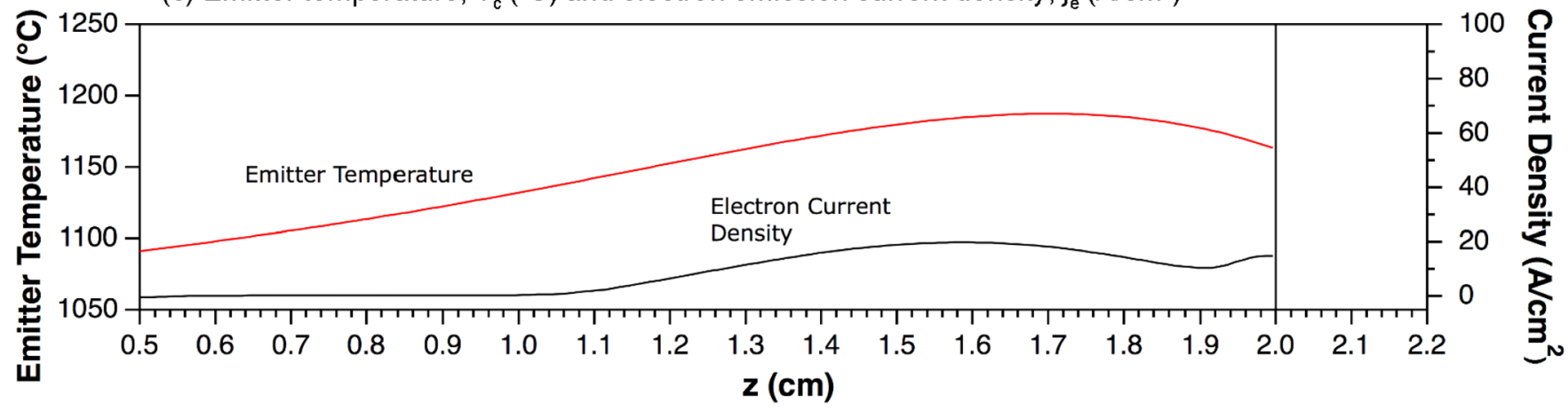

(d) Xenon plasma density contours, $\mathrm{n}_{\mathrm{e}} / 10^{19}\left(\mathrm{~m}^{-3}\right)$ and ion current streamlines.

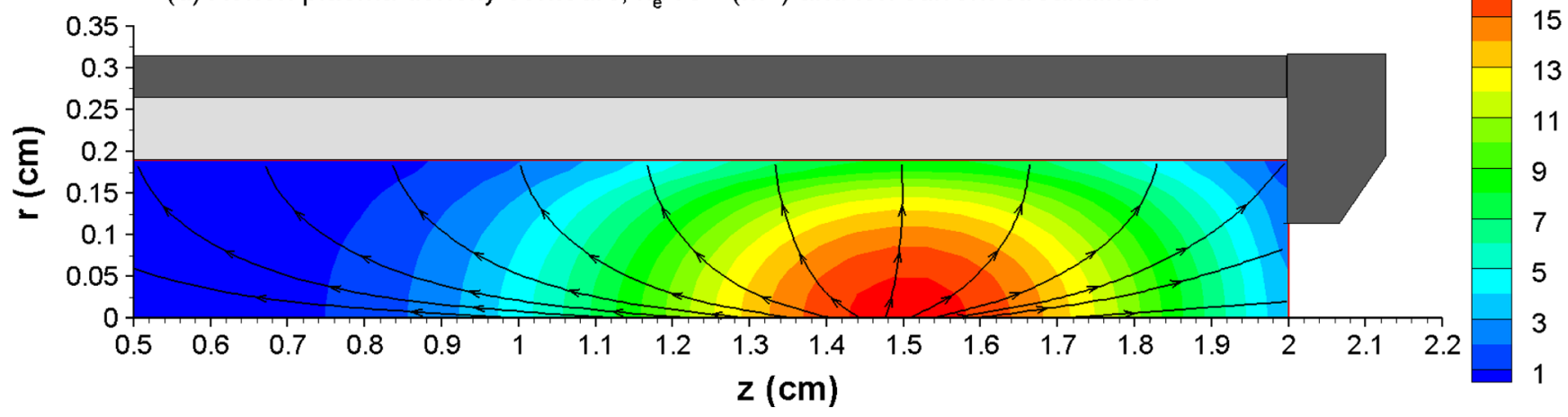

FIG. 3. Xenon plasma solution for the large orifice hollow cathode.

orifice operating at $15 \mathrm{~A}$ and $3.7 \mathrm{sccm}$ are plotted in Fig. 3. Figures 2(a) and 3(a) show the neutral flow field. In the smallorificed cathode, the neutral density is relatively high $\left(\sim 10^{22}\right.$ $\mathrm{m}^{-3}$ ) and drops rapidly in a small region near the orifice because of flow out of the cathode and consumption of neutrals in the strong ionization zone at the downstream end of the insert. The streamlines reveal two sources of neutral gas; the upstream inlet and the return flow of ions neutralized on the walls. The streamlines from both sources converge on the orifice. The xenon neutral density contours in Fig. 3(a) indicate a neutral density about one order of magnitude lower than that in the cathode with the smaller orifice. The low density region at the downstream end also protrudes much further into the insert region than in the smaller orifice cathode. 
Figures 2(b) and 3(b) illustrate the electron current transport as current streamlines and the resultant plasma heating. As shown in Fig. 2(c), the emitter temperature is strongly peaked at the downstream end of the small-diameter cathode and electron emission is confined to a region about $4 \mathrm{~mm}$ long near the orifice plate. The streamlines in Fig. 2(b) show that the electron flow converges on the orifice, and resistive heating from the high current density results in a peak in the electron temperature of about $1.85 \mathrm{eV}$ in the orifice. Further upstream, the plasma potential is low and electrons can penetrate from the plasma to the insert surface, resulting in net electron flow to the insert. Figure 3(c) reveals that the cathode temperature peaks about $3 \mathrm{~mm}$ upstream of the orifice plate in the large orifice cathode and is about $40^{\circ} \mathrm{C}$ lower than in the smaller orifice cathode. The electron emission current density is correspondingly lower and much more broadly distributed, extending over a length of $6-8 \mathrm{~mm}$ at the downstream end of the insert. The current density is peaked $4 \mathrm{~mm}$ upstream of the orifice plate. The electron temperature is considerably higher ( $2.2 \mathrm{eV}$ compared to 1.85 in the smaller orifice cathode), and the broad peak occurs in the interior over the electron emission zone. The region of high electron temperature extends all the way to the wall, where the sheath insulates the surface. Upstream of the domain shown in this plot, the plasma potential drops and there is a net flow of electrons to the insert as observed in the smaller orifice cathode.

The plasma density distribution and resulting ion flow are shown in Figs. 2(d) and 3(d). The high electron temperature in Fig. 2(d) near the orifice leads to a peak plasma density of $\sim 10^{21} \mathrm{~m}^{-3}$ at the downstream end of the insert. Ions created in this ionization zone flow out radially and upstream, as shown by the ion current streamlines. Xenon ions created here are neutralized on the walls and return to the discharge as neutrals, as shown by the neutral flow streamlines in Fig. 2(a). The ion density in the cathode with the larger diameter is about one order of magnitude lower than in the other cathode, and peaks at about $5 \mathrm{~mm}$ upstream of the orifice plate as shown in Fig. 3(d). This coincides with the peak in the emitted electron current density. The ions flow away from the peak, ultimately hitting the walls and returning as neutrals or flowing out of the cathode orifice.

The barium plasma simulation results are shown in Figs. 4 and 5. The mean free path for barium atom ionization $\lambda_{m f p}^{i z}=u_{t, B a} / \nu_{B a}^{i z}$ based on the barium atom thermal velocity $u_{t, B a}=\left(8 k T_{h} / \pi m_{B a}\right)^{1 / 2}$ and the ionization frequency was calculated using the electron density and temperature profiles and is plotted in Figs. 4(a) and 5(a). The mean free path for ionization in the cathode with the smaller orifice is on the order of meters upstream of the dense plasma region, but drops to less than $0.1 \mathrm{~mm}$ near the orifice. Barium atoms that drift into the dense xenon plasma have a high probability of being ionized. The high electron temperatures in the cathode with the bigger orifice creates a large region in which the ionization mean free path is less than $0.1 \mathrm{~mm}$, despite the lower plasma densities as shown in Fig. 5(a).

Figures 4(b) and 5(b) show the neutral barium flow field. Two separatrices are apparent in the flow streamlines plotted for the cathode with the small orifice in Fig. 4(b). The upstream separatrix at an axial location of $0.8-1.1 \mathrm{~cm}$ divides the neutral flow upstream from the neutral flow into the ionization zone. The downstream separatrix divides the flow of neutrals from the insert and the flow of neutrals from the orifice plate. The streamlines also reflect the force balance that results in the steady state neutral density distribution. Xenon ion drag forces are at least two orders of magnitude lower than neutral drag and pressure forces over the entire flow field, so the barium neutral pressure force is balanced by the neutral xenon drag force, $\nabla P_{B a}=\nu_{B a X e} \mu_{B a X e}\left(\mathbf{u}_{B a}-\mathbf{u}_{X e}\right)$. The steep pressure gradient at the edge of the ionization zone downstream is sustained by a high neutral barium diffusion velocity $\mathbf{U}_{B a}=\left(\mathbf{u}_{B a}-\mathbf{u}_{X e}\right)$ and the resulting neutral xenon drag force. At the upstream end, the barium diffusion velocity exceeds the neutral xenon flow velocity, resulting in barium flow upstream. In an intermediate region, where the neutral barium pressure gradient is lower, the neutral barium diffusion velocity required to generate a drag force that balances the pressure force is lower than the xenon flow velocity and the barium flow reverses direction. Between 0.8 and $1.3 \mathrm{~cm}$ at the points where the flow streamlines change direction, the axial barium diffusion velocity is equal to the axial neutral xenon flow velocity of 2-6 m/s. Because of the high probability of ionizing $\mathrm{Ba}$ in the intense Xe plasma located just upstream of the orifice, the Ba neutral density is very low there and little $\mathrm{Ba}$ escapes through the orifice.

The streamlines plotted in Fig. 5(b) for the cathode with the larger orifice indicate that the barium neutrals emitted from the insert and orifice plate flow into the ionization zone. This region of high ionization produces a large region in which barium neutrals are depleted. Barium neutrals also flow upstream, but this occurs upstream of the domain shown in these plots. The steep neutral barium density gradient surrounding the ionization zone is sustained by a balance between the pressure gradient and the drag force associated with collisions between barium atoms and neutral xenon atoms. Because the high density Xe plasma is located further upstream in this cathode, the Ba neutral density is higher near the orifice and more $\mathrm{Ba}$ is lost through the orifice.

The barium ion density distribution and flow streamlines are shown in Figs. 4(c) and 5(c). For the cathode with the small orifice, the barium ion density is near zero inside the dense xenon plasma near the orifice and peaks in a relatively thin ionization front surrounding that zone. Neutral barium flowing toward the intense xenon plasma is ionized at the periphery, and the ions generated in the ionization zone flow outward and ultimately strike the emitter or the orifice plate, where they are neutralized and re-emitted as barium atoms. The steady state barium ion density distribution is the result of a more complex balance of forces. The high density xenon plasma near the orifice has a very low barium ion density because neutrals are ionized before they penetrate very far and the resulting ions are pushed outward by the electric field, which is shown in Fig. 4(d). As a result, very few Ba ions are lost through the orifice. In the region immediately outside the high density xenon plasma, the electric field force is balanced by the barium ion pressure, xenon ion drag, and xenon neutral drag in approximately equal proportions. 
(a) Barium ionization mean free path $(\mathrm{mm})$

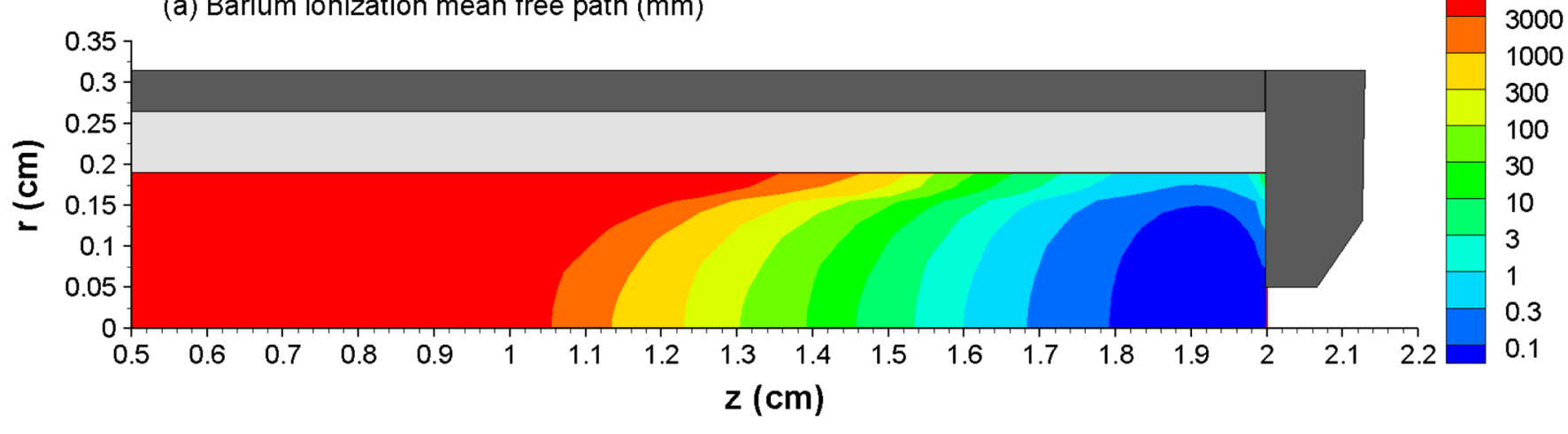

(b) Barium neutral density contours, $\mathrm{n}_{\mathrm{Ba}} / 10^{16}\left(\mathrm{~m}^{-3}\right)$ and barium neutral flow streamlines.

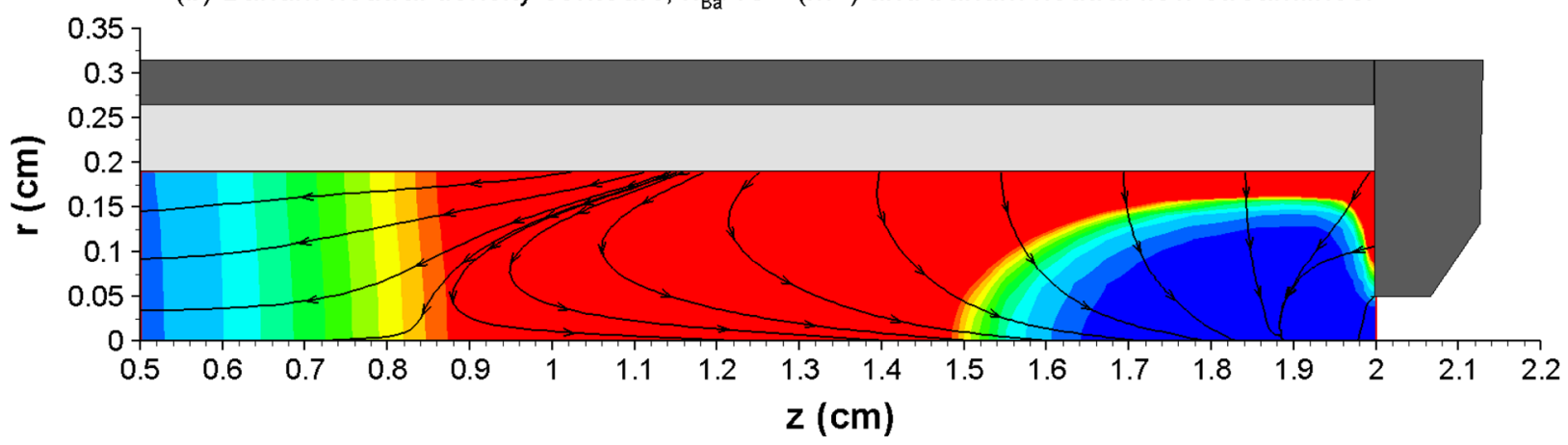

(c) Barium ion density contours, $\mathrm{n}_{\mathrm{Ba}} / 10^{14}\left(\mathrm{~m}^{-3}\right)$ and barium ion flow streamlines.
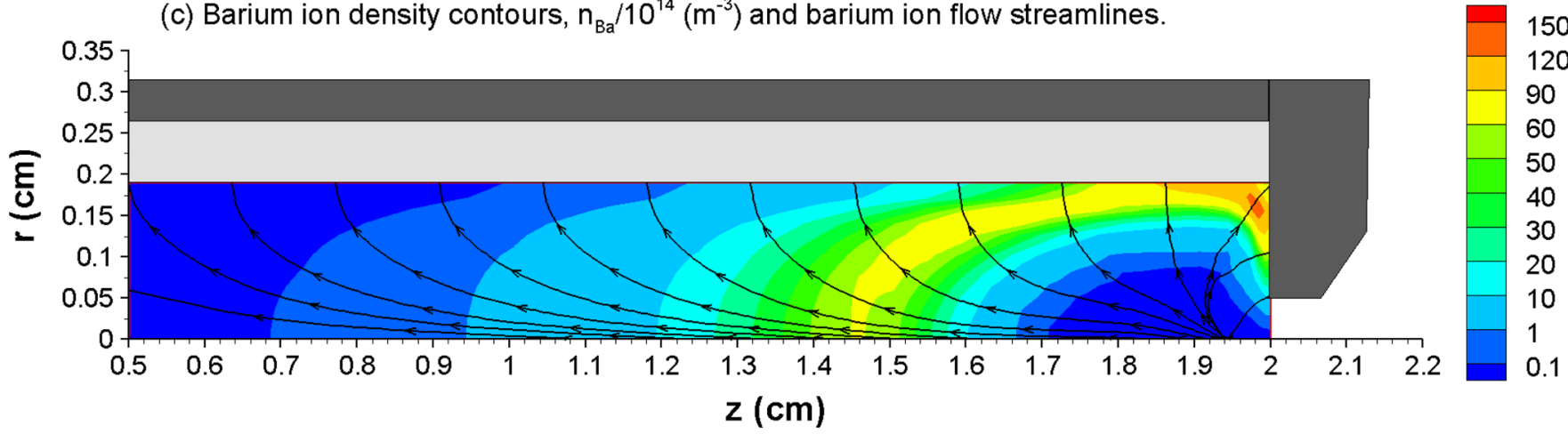

(d) Electric potential contours ( $V$ ) and electric field lines.

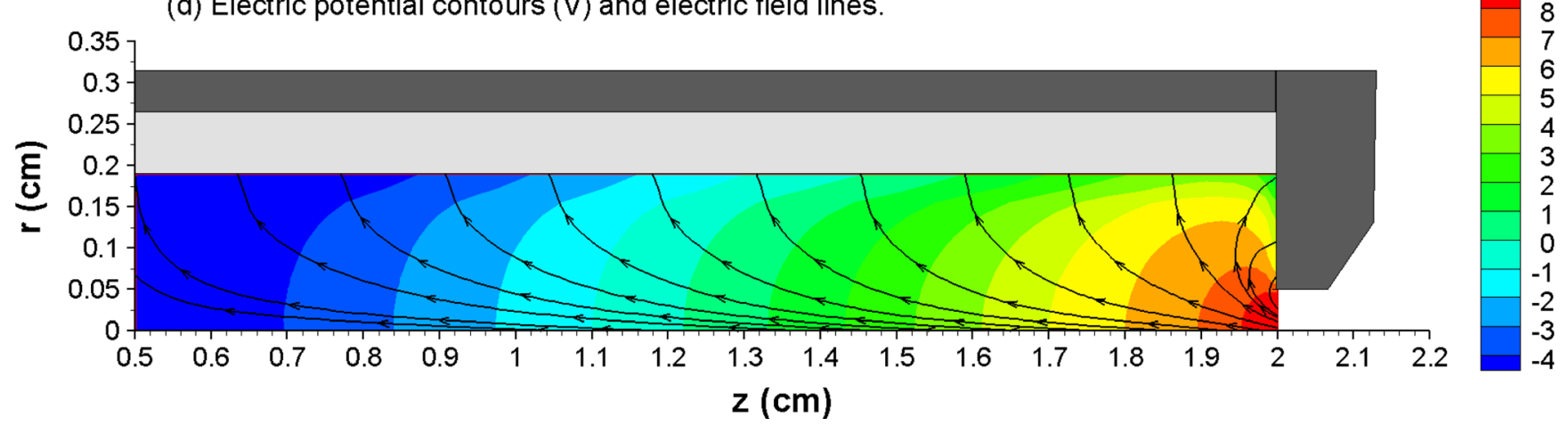

FIG. 4. Barium plasma solution for the small orifice hollow cathode.

Where the barium ion density starts to drop beyond that, the pressure force becomes negligible and the electrostatic force is balanced by ion and neutral drag. At the upstream end of the insert, the xenon ion density drops and the ion drag force become negligible. In this region, neutral drag alone resists the electric field force, although the barium ion density is very low here because most of the ions have been driven to the emitter upstream of that due to strong radial electric fields near the wall.

The barium ion density distribution and flow field shown in Fig. 5(c) is qualitatively similar to that in the small orifice cathode, but is centered over the peak in xenon plasma density in the interior of the cathode. The peak barium ion density here is about one third of the density in the small orifice 
(a) Barium ionization mean free path $(\mathrm{mm})$

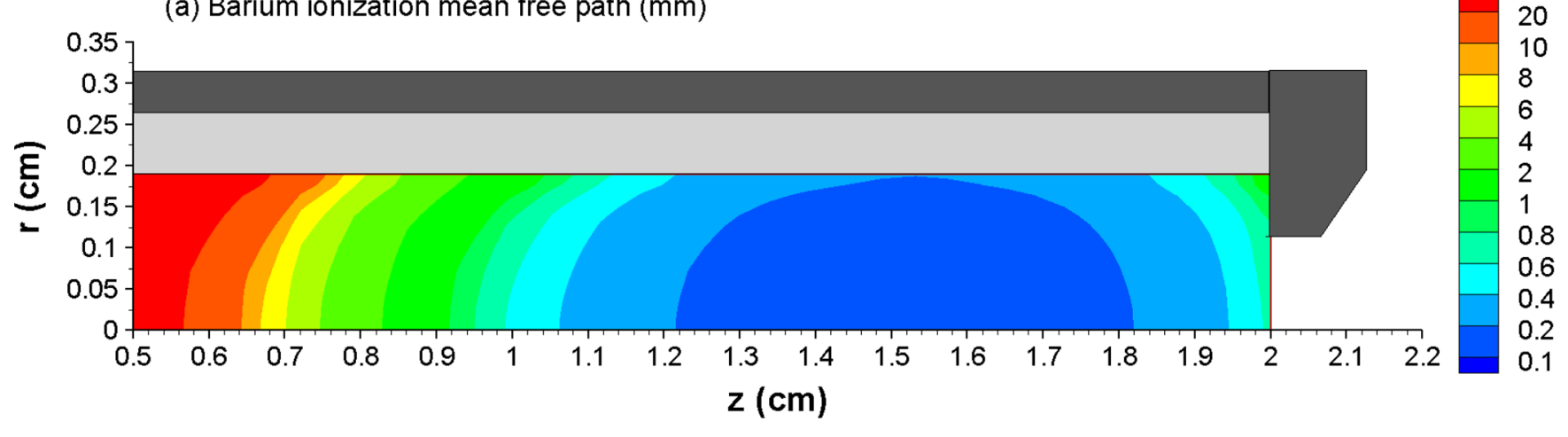

(b) Barium neutral density contours, $\mathrm{n}_{\mathrm{Ba}} / 10^{16}\left(\mathrm{~m}^{-3}\right)$ and barium neutral flow streamlines.

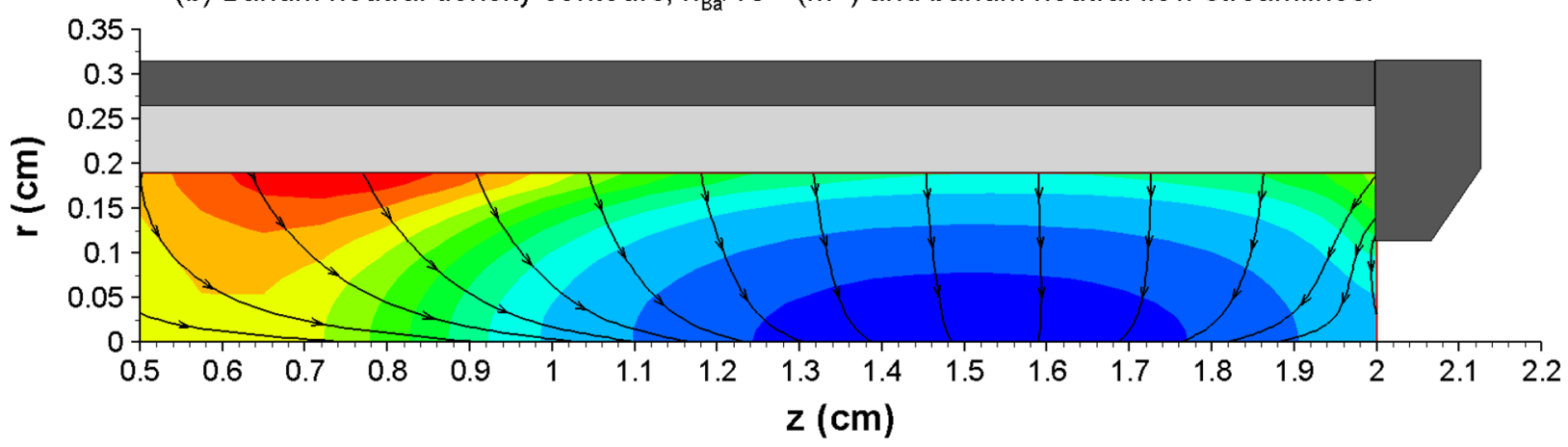

(c) Barium ion density contours, $\mathrm{n}_{\mathrm{Ba}} / 10^{14}\left(\mathrm{~m}^{-3}\right)$ and barium ion flow streamlines.

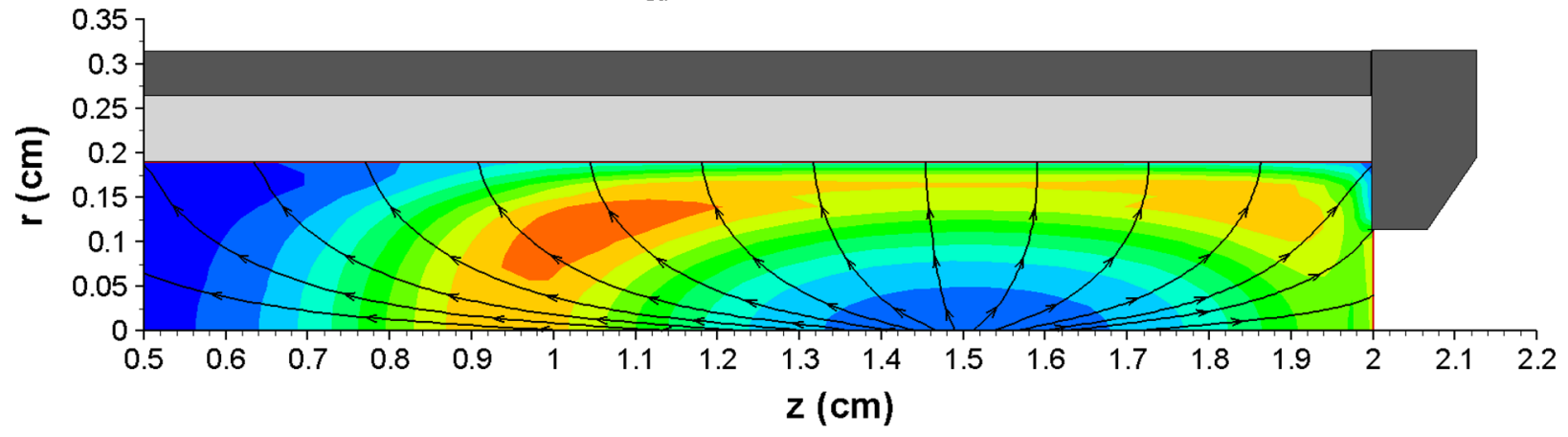

(d) Electric potential contours $(V)$ and electric field lines.

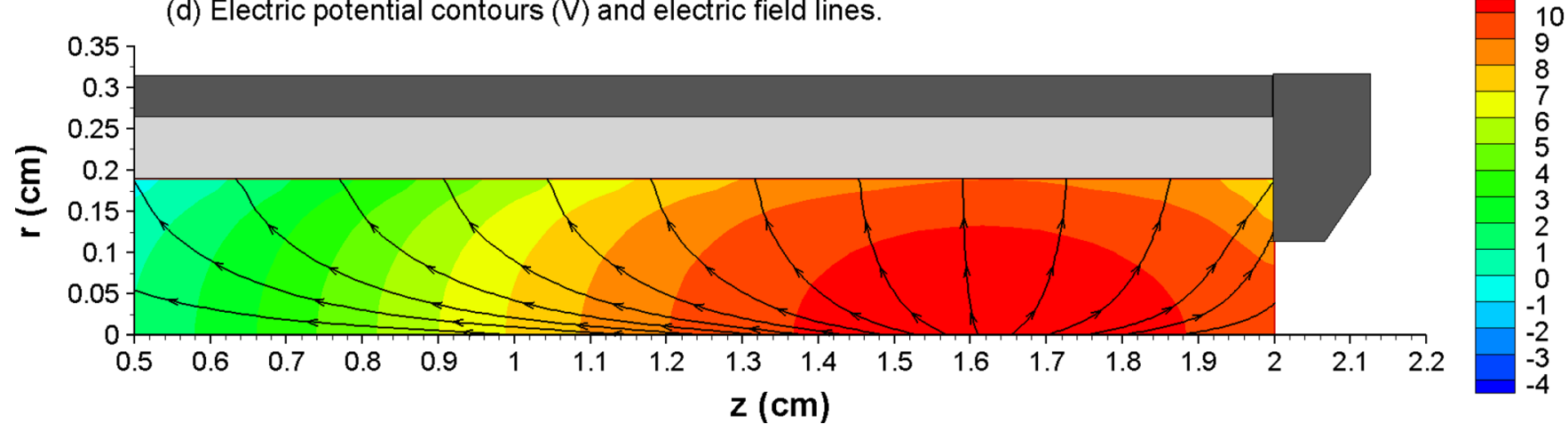

FIG. 5. Barium plasma solution for the large orifice hollow cathode.

cathode. Neutrals that flow toward the ionization zone are ionized in the periphery and the resulting ions are pushed outward by the electric field, shown in Fig. 5(d). Unlike in the small orifice cathode, xenon neutral drag plays very little role in the ion flow dynamics around the ionization zone. In the large orifice cathode, the xenon neutral density is lower by about one order of magnitude, so xenon ion drag is the dominant force opposing the electric field force around the ionization zone. The forces associated with the pressure gradient and neutral drag are five to ten times lower than the other forces. Upstream of the ionization zone, the xenon ion density drops significantly and the electrostatic force is balanced primarily by neutral drag. The strong electric field near the emitter surface causes most of the barium ions 
created in the ionization zone to strike the emitter surface within the first 1 to $1.5 \mathrm{~cm}$, as the contours in Fig. 5(c) show. Because the $\mathrm{Ba}$ ionization region is located further inside the cathode, more $\mathrm{Ba}$ ions are able to escape through the orifice.

The barium neutral pressure at the emitter surface is compared with the barium vapor pressure over the reaction front in the pores of the emitter in Figs. 6(a) and 6(b). Where the neutral pressure in the discharge exceeds the vapor pressure, the reaction in the interior is suppressed and there is no barium flow from the interior. The net barium supply rate in the small orifice cathode is non-zero only over a region about $2 \mathrm{~mm}$ long at the downstream end, as shown in the right hand side of Fig. 6(a). Barium ions are neutralized on the walls and return to the interior as neutrals, providing a second source of neutral gas. There are two sinks for barium neutral gas-the cold surfaces upstream of the emitter, where barium condenses and the ionization zone, where neutrals are consumed in ionizing reactions. The neutral density is so low near the orifice that the neutral loss rate through the orifice is negligible. In the larger orifice cathode, the orifice is a third sink for $\mathrm{Ba}$. The additional sink and the large ionization zone result in a Ba neutral pressure about one order of magnitude lower over most of the insert compared to the distribution in the small orifice cathode. The vapor pressure at the reaction front exceeds the $\mathrm{Ba}$ pressure in the insert plasma over the first $\mathrm{cm}$ of the insert, leading to net $\mathrm{Ba}$ flow from the insert interior over a much broader area than in the small orifice cathode.

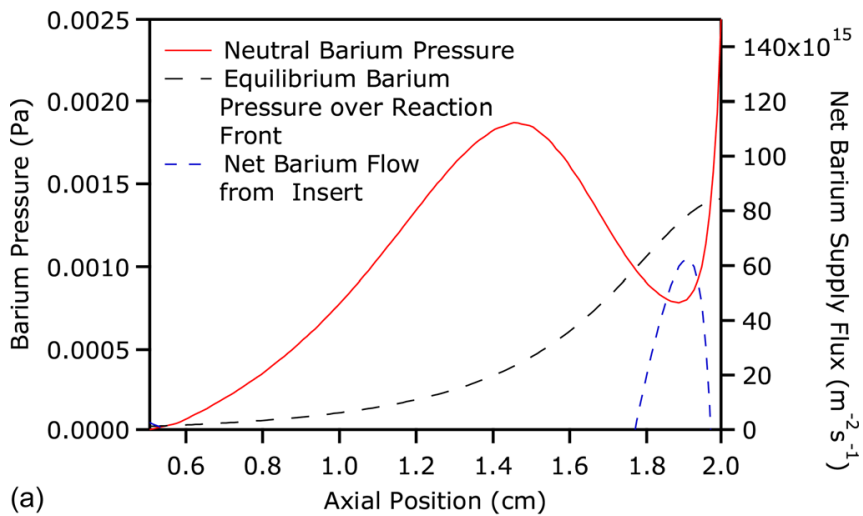

(a)

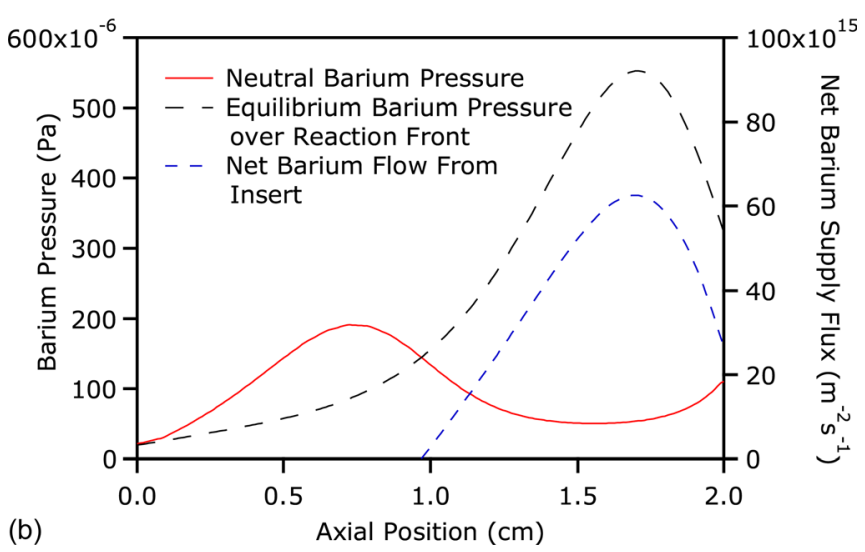

FIG. 6. Barium partial pressure, vapor pressure over the reaction front, and net supply rate for the small orifice cathode.
In steady state, the net Ba flow from the insert must balance the losses to surfaces upstream of the insert and through the orifice. In the small orifice cathode $63 \%$ of the barium that escapes flows upstream of the simulation domain (primarily as neutrals), with the remainder flowing through the orifice as ions. In the large orifice cathode, the loss of ions through the orifice is larger, representing $65 \%$ of the total loss. The remaining $\mathrm{Ba}$ loss upstream of the simulation domain is largely in the form of ions, because the ionization zone extends further back in this cathode. As a result of greater $\mathrm{Ba}$ losses in the larger orifice cathode, it consumes $\mathrm{Ba}$ at a rate five times higher than that of the cathode with the smaller orifice, even though the cathode temperature is lower.

\section{EFFECT ON EMITTER SURFACE COVERAGE}

The Ba surface coverage that can be maintained with resupply from the gas phase is estimated using a surface kinetics model. Neglecting the contribution from surface diffusion out of the pores in the tungsten and assuming there are no weakly bound precursor states for adsorbed $\mathrm{Ba}$, the time rate of change of $\mathrm{Ba}$ atom density on the surface can be expressed as

$$
N_{0} \frac{\partial \theta}{\partial t}=\left(\Gamma_{B a}+\Gamma_{B a^{+}}\right)(1-\theta)-\Gamma_{d}
$$

In this expression, $N_{0}=5 \times 10^{18} \mathrm{~m}^{-2} \mathrm{~s}^{-1}$ is the number density of adsorption sites for $\mathrm{Ba}$ on tungsten, ${ }^{15} \theta$ is the coverage, or fraction of these sites that are occupied, and $\Gamma_{B a}$ and $\Gamma_{B a+}$ are the fluxes of $\mathrm{Ba}$ atoms and ions to the surface. The sticking coefficient of $\mathrm{Ba}$ on tungsten is assumed to be unity, so the probability that impinging barium particles will adsorb is proportional to the fraction of open adsorption sites, $(1-\theta)$.

The Ba desorption rate, $\Gamma_{d}$, can be described by an Arrhenius relationship, $\Gamma_{d}\left(T_{c}, \theta\right)=N_{0} \nu_{d}(\theta) \exp \left(e E_{d}(\theta) / k T_{c}\right)$. The desorption energy $E_{d}$ and pre-exponential factor $\nu_{d}$ are both strong functions of $\theta$ because of adatom interactions. At high coverage, interactions between neighboring adatoms reduce the binding forces, making it easier for adatoms to thermally desorb. This can be modeled semi-empirically assuming a constant desorption energy and a pre-exponential factor that varies as $\theta^{5}$. Using desorption data for Ba on oxidized $\mathrm{W},{ }^{15}$ an expression for the desorption flux in this form can be derived

$$
\Gamma_{d}=1.05 \times 10^{25} \theta^{5} \exp \left(-2.06 e / k T_{c}\right) .
$$

Under steady state conditions, the equilibrium surface coverage is given by a balance between the supply rate from the gas phase and loss by desorption

$$
\frac{1-\theta}{\theta^{5}}=\frac{1.05 \times 10^{25} \exp \left(-2.06 e / k T_{c}\right)}{n_{B a} u_{t, B a}+n_{B a+} u_{B}} .
$$

For the conditions described here, the ion flux to the emitter is much higher than the neutral flux. Figs. 7(a) and 7(b) show the ion fluxes and resulting surface coverages. For the small orifice hollow cathode, the ion flux is strongly peaked at the 

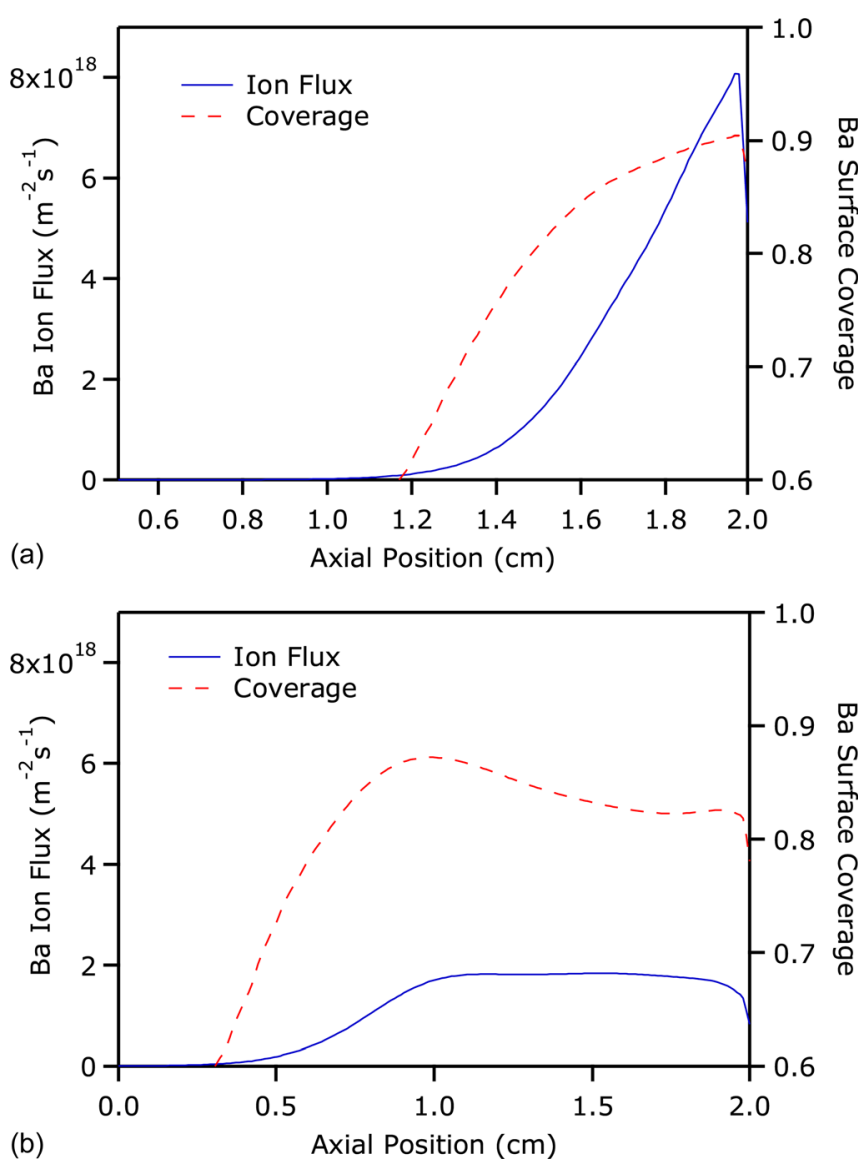

FIG. 7. Barium ion flux and resulting surface coverage.

downstream end, but yields a surface coverage greater than 0.8 over the first $4 \mathrm{~mm}$ of the emitter. For the larger orifice cathode, the ion flux is lower, but is spread over a much larger length on the insert and maintains a surface coverage greater than 0.8 over almost $1.5 \mathrm{~cm}$ of the emitter. These coverage levels are more than adequate to support the calculated current densities. The earlier study ${ }^{5}$ demonstrated that these high coverage levels can be maintained over tens of thousands of hours of operation. The ion flux drops as the supply rate decreases due to recession of the reaction front in the insert, but the coverage is very weakly dependent on the ion flux, as shown in Eq. (7).

\section{CONCLUSIONS}

The simulations of these two cathode geometries reveal several important aspects of Ba transport. First, despite large differences in the internal Xe plasma conditions and emitter operation, Ba flow dynamics are qualitatively very similar. The small and large orifice cathodes have Xe neutral and ion densities that differ by an order of magnitude, and the electron temperature varies by $20 \%$. The area over which the plasma contacts the emitter differs by a factor of three, resulting in about a factor of four differences in electron emission current density and a $40{ }^{\circ} \mathrm{C}$ difference in peak emitter temperature. In both cases, neutral $\mathrm{Ba}$ produced by the insert is ionized and recycled back to the emitter surface, where it is needed in the emission zone. In addition, the high partial pressure of $\mathrm{Ba}$ in the insert plasma suppresses the production of $\mathrm{Ba}$ in the insert over a significant fraction of its length.

However, comparison of the two geometries shows that cathode design can have a significant quantitative effect on barium loss rates. The small orifice cathode design results in a larger xenon pressure and a concentration of the xenon plasma near the orifice. Barium is very effectively confined to the cathode interior because it is ionized in this dense plasma and pushed away from the orifice. Despite significantly lower current density and temperature, $\mathrm{Ba}$ is consumed five times faster in the large orifice cathode because the xenon plasma density is much lower near the orifice than in the small orifice cathode. This highlights the fact that the Ba dynamics should be assessed in every new cathode design and that design attributes which seem beneficial (such as lower current density and temperature) can result in plasma distributions that do not contain $\mathrm{Ba}$ as effectively.

Finally, the dynamics that establish the Ba flow field and density distribution are more complex than previously realized. $\mathrm{Ba}$ is not simply swept out of the cathode with the Xe gas flow, nor is it pushed upstream away from the emission zone by the axial electric field. The majority of the neutral Ba produced by the emitter flows into the ionization zone at the periphery of the dense Xe plasma and is consumed by ionization reactions. A pressure gradient balanced by neutral Xe drag results in a neutral density distribution peak at the insert surface and a sharp gradient into the Xe plasma core, where the density is 5-7 orders of magnitude lower. The $\mathrm{Ba}^{+}$ion motion is largely determined by the electric field, which forces ions out of the ionization zone to the insert surface and to the orifice plate in the small orifice cathode or out of the orifice as well as upstream in the larger cathode. The $\mathrm{Ba}^{+}$ion density outside the ionization zone initially increases radially, and the electric field force is opposed by the pressure gradient and drag forces (ion and neutral drag in the small orifice cathode and ion drag alone in the large orifice cathode because the $\mathrm{Xe}$ neutral density is so low). Closer to the emitter, the ion density drops radially as the strong electric field near the surface accelerates the ions, and drag forces dominate over pressure gradient forces in balancing the electrostatic force. The emitted barium ultimately leaves either through the upstream end of the insert, where it condenses on colder surfaces in the cathode assembly or downstream through the orifice.

Because of these processes, hollow cathodes are much more complex than vacuum cathodes employing similar emitters. The dynamics of barium transport in the internal hollow cathode plasma can be exploited successfully with modeling tools like those reported here to extend cathode life.

\section{ACKNOWLEDGMENTS}

The research described in this paper was carried out by the Jet Propulsion Laboratory, California Institute of Technology, under a contract with the National Aeronautics and Space Administration. 
${ }^{1}$ P. Palluel and A. Shroff, J. Appl. Phys. 51, 2894 (1980).

${ }^{2}$ T. Sarver-Verhey, in Proceedings of $34^{\text {rd }}$ Joint Propulsion Conference (Cleveland, OH, 1998), AIAA-98-3482.

${ }^{3}$ J. Polk, J. Anderson, J. Brophy, V. Rawlin, M. Patterson, and J. Sovey, in Proceedings of $35^{\text {th }}$ Joint Propulsion Conference (Los Angeles, CA, 1999), AIAA-99-2446.

${ }^{4}$ A. Sengupta, J. Brophy, and K. Goodfellow, in Proceedings of $40^{\text {th }}$ Joint Propulsion Conference (Fort Lauderdale, FL, 2004), AIAA-2004-4558.

${ }^{5}$ J. Polk, I. Mikellides, I. Katz, and A. Capece, J. Appl. Phys. 105, 113301 (2009).

${ }^{6}$ G. Gaertner, P. Geittner, D. Raasch, and D. Wiechert, Appl. Surf. Sci. 146, 22 (1999).

${ }^{7}$ I. Mikellides, I. Katz, D. Goebel, and J. Polk, J. Appl. Phys. 98, 113303 (2005).
${ }^{8}$ T. G. Walker, K. Bonin, and W. Happer, J. Chem. Phys. 87, 660 (1987).

9. Dettmann and F. Karstensen, J. Phys. B: At. Mol. Phys. 15, 287 (1982).

${ }^{10}$ A. Sengupta, in Proceedings of $29^{\text {th }}$ International Electric Propulsion Conference (Princeton, NJ, 2005), IEPC 2005-026.

${ }^{11}$ E. Rittner, W. Rutledge, and R. Ahlert, J. Appl. Phys. 28, 1468 (1957).

${ }^{12}$ A. Shroff, P. Palluel, and J. Tonnerre, Appl. Surf. Sci. 8, 36 (1981).

${ }^{13}$ J. Polk, C. Marrese-Reading, B. Thornber, L. Dang, L. Johnson, and I. Katz, Rev. Sci. Instrum. 78, 093101 (2007).

${ }^{14}$ A. Sengupta, J. Kulleck, N. Hill, and W. Ohlinger, Rev. Sci. Instrum. 79, 111301 (2008).

${ }^{15}$ R. Forman, J. Appl. Phys. 47, 5272 (1976). 